\title{
A population study of Norwegian psychiatric patients referred for clinical brain scanning
}

\author{
Mona K. Beyer, Turi O. Dalaker, Ole J. Greve, Siv E. Pignatiello and Ingrid Agartz
}

\section{Background}

Patients with psychiatric conditions are often referred for a brain scan during the course of their diagnostic workup.

\section{Aims}

The aim of our study is to determine frequency and type of organic brain pathology, the relationship to age, gender and psychiatric diagnosis.

\section{Method}

We investigated magnetic resonance imaging and computed tomography brain scans from consecutively referred patients over a 10-year period (January 2002-December 2011). The reasons for referral, estimated psychiatric diagnosis, and the pathology discovered for each patient were registered.

\section{Results}

A total of $34 \%$ of patients demonstrated organic brain pathology, of which $32.8 \%$ were considered clinically relevant. This represents a higher frequency of relevant pathology than reported in healthy subjects. Age $(P<0.001)$ and diagnosis $(P=0.016)$ were the most important determinants for frequency of pathological findings.

Computed tomography and magnetic resonance imaging (MRI) of the brain are diagnostic complements in the investigation of patients with psychiatric symptoms that may have an underlying organic brain disease. ${ }^{1}$ The knowledge about the frequency and type of positive brain findings resulting from these undertakings is limited.

\section{Brain pathologies in psychiatric disease}

The prevalence of organic brain pathology is found to be increased in past studies in some psychiatric samples, ${ }^{1}$ although others have not found the same in more recent studies. ${ }^{2}$ Tumours, infarcts or encephalitis may present with psychiatric manifestations that vary widely across the neuropsychiatric spectrum, but organic pathology also commonly features in individuals with psychiatric disorders. Developmental brain abnormalities appear more commonly in genetic or toxic brain syndromes (e.g. schizophrenia, intellectual disability and foetal alcohol syndrome) and in children with developmental delay of unknown etiology. ${ }^{3}$ White matter lesions (WMLs) are found in old-age depression ${ }^{4}$ and occur in adolescent bipolar and unipolar depressive disorder. ${ }^{5}$

\section{Study design of previous studies}

Ideally, to obtain prevalence rates in the psychiatric population, screening of large population-based unselected samples at different ages should be undertaken. To our knowledge, there are no results available from large population-based studies of psychiatric populations, but there are results available from clinical psychiatric cohorts $^{6}$ and smaller population-based studies. ${ }^{2,7}$ There are two similar studies available, the first of which was carried out in 19941998, with 435 in-patients aged 16-65 years. ${ }^{7}$ In this retrospective study, ${ }^{7}$ the referral practice, outcome and predictive factors for abnormal scans were investigated. The authors had access to the medical

\section{Conclusions}

Brain imaging in clinical psychiatry resulted in approximately $30 \%$ positive findings mainly associated with increasing pathologies with age, but also with diagnosis.

\section{Declaration of interest}

Both T.O.D. and M.K.B. have received honorary from Novartis for scientific lectures about multiple sclerosis. M.K.B. also received honoraria from Biogen for scientific lectures. The other authors have no conflicts of interest.

\section{Copyright and usage}

(C) The Royal College of Psychiatrists 2018. This is an Open Access article, distributed under the terms of the Creative commons Attribution-NonCommercial-NoDerivatives licence (http://creativecommons.org/licenses/by-nc-nd/4.0/), which permits noncommercial re-use, distribution, and reproduction in any medium, provided the original work is unaltered and is properly cited. The written permission of Cambridge University Press must be obtained for commercial re-use or in order to create a derivative work.

files and $14.3 \%$ of the patients had abnormal scans. The second study included 656 patients with psychosis. ${ }^{2}$ The radiological reports were studied and classified as either normal, non-relevant abnormalities or clinically relevant brain abnormalities. Clinically relevant pathology was found in $11.1 \%$ of the patients.

The structural brain changes commonly found in psychiatric or neuropsychiatric disorders are typically discrete and unspecific. There is a large overlap with brain structure variation found in healthy individuals, and such changes are not diagnostic in the individual patient. ${ }^{8}$ Still, organic brain pathology can present with only psychiatric symptoms in a number of diseases including encephalitis, tumours, multiple sclerosis and neurosarcoidosis. ${ }^{9}$ In clinical practice, psychiatric patients are therefore often referred for a brain computed tomography scan or MRI on wide indications.

We conducted a retrospective study of all clinical patients from the Division of Psychiatry at Stavanger University Hospital, Stavanger, Norway, referred for brain scanning within a 10 -year period.

The aim of the study was to determine the frequency and type of organic brain pathology, and the relationship to age, gender and psychiatric diagnosis. To our knowledge, this kind of study has not been conducted previously. We hypothesised that patients referred from psychiatric clinics have a higher frequency of organic brain pathology than reports of healthy patients of similar age, and that frequency and type of brain pathology are related to psychiatric diagnosis and age.

\section{Method}

\section{Participants}

We collected anonymised information from the radiology information system (RIS) from the only public hospital in the Stavanger 
region. The RIS is a computerised database used by the hospital's radiology department to store radiological data from all radiological examinations. All consecutive referrals from the Division of Psychiatry, Stavanger University Hospital, to either a computed tomography or MRI brain scan at the radiology department were included. The referrals included in- and out-patients from all clinics associated with the psychiatric division.

\section{Data collection}

A total of 3614 patients were referred over the 10 -year study period and all referrals were included. Information on patients' age at the time of referral, gender, type of scan (computed tomography or MRI), cause of referral/indication, referral text, scan date and the written report by the radiologist was systematically collected. The written radiologist report was reviewed to determine the type of brain pathology.

\section{Data flow}

Out of the total number of 3614 referrals, 2922 patients completed a brain scan. For details on data flow see Supplementary Figure 1, available at https://doi.org/10.1192/bjo.2018.16.

\section{Referral categories}

Referrals from the 2922 patients who completed a brain scan were then categorised into three groups by two specialists in radiology (T.O.D. and O.J.G.). The first group was routine referrals $(n=$ 2563). The category 'routine' was used for patients where the referral text clearly expressed that the scan was scheduled as part of a work-up for psychiatric symptoms. In these referrals, no specific suspicion of brain pathologies was expressed by the referring physician. The second group was 'to exclude cerebral tumour' $(n=127)$. This category necessitated a clear clinical suspicion, or signs/symptoms of brain tumour in the referral text. The third group was 'specific clinical question' $(n=232)$, including follow-up examinations, e.g. after surgery, treatment of pituitary adenomas or post-trauma, as formulated by the referring physician. The categorisation of patients was done for consecutive referrals and all the referrals were included.

\section{Age categories}

The sample was divided into age decades to show changes with age.

\section{Psychiatric diagnostic categories}

The psychiatric diagnosis was assessed by an experienced specialist in clinical psychiatry (S.E.P.), who diagnosed each participant based on ICD-10 criteria, ${ }^{10}$ with the information given in the electronic referral form. Fifteen different diagnostic categories were used; see Supplementary Table 1 for details.

\section{Brain pathology categories}

Brain pathologies were categorised as presented in Table 1. The category 'other pathologies' includes post-operative changes, anomalies and changes secondary to trauma. It is common to rate incidental findings into significant abnormalities, requiring referral or not significant abnormalities as defined by Sommer et al. ${ }^{2}$ The pathologic findings in this study were also rated as either clinically relevant or non-relevant, as has been done in several studies of incidental findings in healthy populations. ${ }^{11}$ Clinically relevant scans are categorised as scans needing routine, urgent or immediate referral. Sommer et al. published lists of all radiological findings, which we used and then classified them as either not clinically relevant or clinically relevant according to that. ${ }^{2}$

We evaluated occurrence of tumours in two of the referral categories: routine $(n=2563)$ or to exclude cerebral tumour $(n=127)$. Intracranial tumours were divided into intra-axial tumours, which are parenchymal tumours, whereas extra-axial tumours are outside the brain parenchyma but inside the skull, e.g. meningioma. ${ }^{12}$

\section{Interrater reliability}

Before the rating work was started, the radiologists went through several sessions of training. Interrater reliability was established with Cronbach's alpha for clinical indication and the reported brain pathologies. Reliability for indications had a Cronbach's alpha of 0.837 , and reliability for pathologies had a Cronbach's alpha of 0.844 . Both reliability analyses were found to be good $(>0.8) .{ }^{13}$

\section{Ethical permission}

The Western Norway Regional Committee for Medical Research Ethics considered the study to be a quality assurance study regarding the use of computed tomography/MRI in psychiatric diseases and therefore it did not require ethical approval. All data were anonymised before we received them. The Privacy and Data Protection Officer in Stavanger University Hospital (Intern ID 2012/3) and the Southeastern regional health authority, Norway (2011/2460 C) approved the study.

\section{Data analyses}

We counted all reported pathologic brain findings in the population. In patients with multiple brain pathologies, we used the

\begin{tabular}{|c|c|c|c|c|c|}
\hline Diagnosis & Number of patients & Routine referral, \% & Mean Age (s.d.) & Gender, M/F \% & Pathology, \% \\
\hline Confusion/dementia & 557 & 93 & 73.9 (13.2) & $40 / 60$ & 77.2 \\
\hline Head trauma & 12 & 100 & $58.2(24.1)$ & $50 / 50$ & 66.7 \\
\hline Other somatic/organic disease & 140 & 80 & $48.7(23.4)$ & $52 / 48$ & 44 \\
\hline Affective depression & 390 & 94 & $53.0(23.2)$ & $38 / 62$ & 32.3 \\
\hline Affective bipolar & 195 & 95 & $46.1(20.6)$ & $42 / 58$ & 26.2 \\
\hline Anxiety (including PTSD) & 19 & 95 & $36.1(22.4)$ & $32 / 68$ & 15.8 \\
\hline Other & 195 & 98 & $23.5(20.0)$ & $56 / 44$ & 15.9 \\
\hline Psychosis & 779 & 97 & $34.9(18.8)$ & $56 / 44$ & 15.3 \\
\hline Substance misuse, not alcohol & 16 & 100 & $28.6(9.8)$ & $69 / 31$ & 12.5 \\
\hline Autism spectrum disorder, Asperger & 180 & 99 & $9.3(4.7)$ & $81 / 19$ & 7.7 \\
\hline Anorexia, eating disorder & 14 & 93 & $16.4(1.8)$ & $0 / 100$ & 7.1 \\
\hline ADHD, Tourette & 187 & 99 & $13.0(4.9)$ & $70 / 30$ & 4.3 \\
\hline Alcohol misuse & 6 & 100 & $58.5(5.2)$ & $83 / 17$ & 0 \\
\hline Whole sample & 2690 & & $43.1(26.8)$ & $51 / 49$ & 31.8 \\
\hline
\end{tabular}


main pathologic finding. We divided the frequency of pathologies according to age groups, type of referral, one of three age groups, age decades and psychiatric category.

To investigate the relationship between pathologies and diagnosis and pathologies and age, correlation analyses with Kendall rank correlation coefficient was used, and multiple regression analysis was performed with pathologies as the dependent variable and age, diagnosis, and age $\times$ diagnosis as predictors. Because of multicollinearity between diagnosis and age, we used centred variables for diagnosis and age. For all statistical analyses, we used IBM SPSS statistics (version 21.0, release 21.0.0.2). Significance level was defined as $P<0.05$.

\section{Results}

\section{Computed tomography and MRI scanning in the study period}

The 2922 brain scans were completed in the period January 2002December 2011. The number of computed tomography and MRI scans increased from 114 in 2002 to 266 scans in 2011. For distribution of computed tomography and MRI examinations conducted yearly during the study period, see Supplementary Figure 1. Routine referrals varied from 80 to $100 \%$ across the diagnostic groups (Table 1).

\section{Frequency of brain pathology in the whole sample}

In the whole population $(N=2922), 34.3 \%$ of scans demonstrated pathologic findings. $3.3 \%$ of all patients had two pathologic findings on their scans and $0.8 \%$ had more than two pathologic findings. After removing all referrals with a specific clinical question $(n=$ 232) the overall percentage of pathologies was reduced to $31.7 \%$ (see Supplementary Table 2). In our study, 32.8\% of reported pathologies were considered to be clinically relevant, whereas only $1.3 \%$ were not considered clinically relevant according to previous published categories. ${ }^{2}$

\section{Frequency according to modality (computed tomography and MRI)}

We looked at the percentage of pathologies found on computed tomography scans versus MRI scans. An interesting finding is that MRI was used more frequently to scan patients under 20 years of age, but after that, computed tomography was more commonly used for all ages. The highest ratio of computed tomography scanning versus MRI scanning was found in the oldest age group, where computed tomography was used 3.6 times as often as MRI. An overview of reported pathologies on computed tomography scans versus MRI scans are found in Table 2. In general, it shows that pathologies such as WMLs and atrophy are more often reported in MRI scans than computed tomography scans. A total of $37 \%$ of patients scanned with MRI had clinically relevant pathologies, whereas patients scanned with computed tomography had 30\% clinically relevant pathologies. This could imply that MRI is more likely than computed tomography to pick up clinically relevant pathology, which may alter the clinical follow-up and treatment of the patient.

\section{Brain pathology in relation to psychiatric diagnosis}

The most frequent overall pathology was WMLs, which was the most frequent pathology in the affective depression, affective bipolar, confusion/dementia, psychosis, ADHD, head trauma and other diagnosis categories. The second most common pathology was atrophy, which was also most frequently found in the confusion/dementia, psychosis and two depression groups.

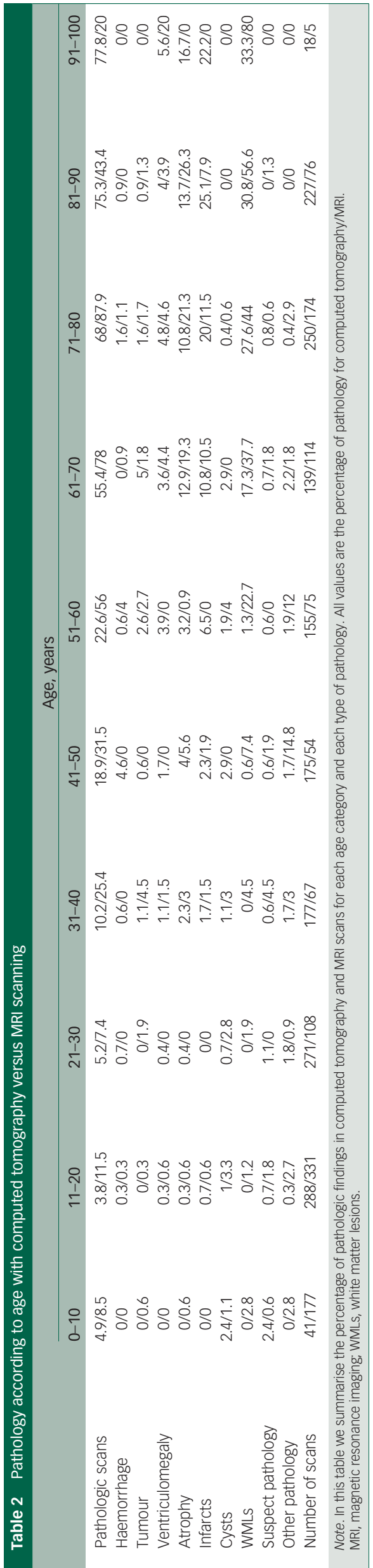


Infarcts were the third most common pathology, found most frequently in confusion/dementia, psychosis and affective depression. Of interest is the finding that more cysts were found in the psychosis group, with $1.9 \%$ of patients reporting with cysts as the main pathology. Ten out of 15 cysts were arachnoid cysts. Two females had pineal cysts, two patients had neuroepithelial cysts and two had other cysts.

Table 1 presents an overview of the percentage of pathological findings in the different diagnostic categories, and Table 3 details the different pathologies in each diagnostic category.

Age and type of pathology was significantly correlated $(r=$ $-0.567, P<0.001)$, as were diagnosis and pathology $(r=0.234$, $P<0.001)$ and diagnosis and age $(r=-0.504, P<0.001)$. An interaction variable was calculated as the product of age $\times$ diagnosis. When using a regression model with age, diagnosis and the interaction variable as predictors, age $(P<0.001)$ and diagnosis $(P=0.016)$ were significantly associated with pathology, but the interaction variable was not significant $(P=0.078)$.

\section{Brain pathology in relation to age}

Mean age of the whole population was 44.1 years (range: 2-96 years). There were two age peaks with more patients scanned at age groups $14-20$ years and $65-85$ years. A total of $49 \%$ of participants were women $(n=1434)$. Before 30 years of age, over $90 \%$ of brain scans had no pathologies, whereas in the age group 91-100 years, only $17.4 \%$ were reported as negative scans.

WMLs were the most frequently found pathology, reported on $12.8 \%$ of all scans. Brain atrophy and brain infarcts were the second and third most prevalent pathologies. These pathologies increased with increasing age, except for the oldest age group where atrophy and infarcts were slightly less prevalent than in the age group 8090 years, relative to the number of individuals in the group. Ventriculomegaly was found with increasing age, also in the oldest patients (age 91-100 years). Extra-axial tumours increased with age, whereas intra-axial tumours did not. Please see the distribution of pathological findings per age decade in Table 4 and Fig. 1.

There were 29 tumours among patients referred for routine reasons $(n=2563)$ or to exclude brain tumour $(n=127)$. In the routine referrals group, we found 15 extra-axial $(0.6 \%)$ and seven intra-axial tumours $(0.3 \%)$. In the referrals to exclude brain tumour group, we found two extra-axial tumours (1.6\%) and five intra-axial tumours (3.9\%).
The occurrence of tumours varied from $0.2 \%$ of patients in the age group $11-20$ years to $3.2 \%$ in the age group $61-70$ years. The most frequent tumours were meningiomas $(n=16$, age range: 43-85 years).

\section{Discussion}

The main finding of this study is that $32 \%$ of brain scans from a large, pre-selected clinical population referred from a psychiatric department demonstrated pathological findings. In a regression model, age and diagnosis were the factors most significantly associated with pathology. We found a significant correlation between both diagnosis and age for all types of pathologies, but in the multiple regression analysis, age was the most important parameter to explain increased pathologies in our cohort, and diagnosis was the second most important predictor. Sommer et al. also found age to be the best predictor of brain pathology in their study of patients with psychotic episodes, ${ }^{2}$ although there was no significant effect of diagnosis on the distribution of pathologies, which is different from our results. However, Sommer et al. only studied patients with psychosis and had a smaller cohort, ${ }^{2}$ which may explain the difference. In a large cohort study of psychosis patients at different illness stages by Lubman et al., ${ }^{14}$ incidental findings were also found to be increased with age.

In patients younger than 20 years of age, the frequency of pathological findings was $7 \%$, whereas another study of healthy youths by Gur et al. reported a frequency of $10 \%$ incidental findings. ${ }^{15}$ In one study by Kim et al. of 225 healthy children, $21 \%$ of children volunteers had unexpected findings of which as many as $36 \%$ required routine referral for further evaluation. ${ }^{11}$ It is surprising that we find lower frequency of pathologies in our cohort than in studies of healthy children. The discrepancy between these studies could be because of methodological differences, as one study ${ }^{11}$ systematically reviewed the scans whereas we reviewed the radiology reports but not the images. Another possibility is that children with clinical/neurological symptoms in our hospital may have already been referred by paediatricians or other clinicians, and that the remaining children are referred from the division of psychiatry and will subsequently have lower frequency of pathologies.

In adults aged 18-65 years, we found an overall pathology of $21 \%$, whereas in a study by Illes et al. ${ }^{16}$ a prevalence of $15 \%$ in 151 apparently normal adult control participants was reported. In

\begin{tabular}{|c|c|c|c|c|c|c|c|c|c|c|}
\hline Diagnosis & Haemorrhage & Tumour & Ventriculomegaly & Atrophy & Infarcts & Cysts & WMLS & $\begin{array}{l}\text { Suspect } \\
\text { pathology }\end{array}$ & Other & Patients \\
\hline Alcohol misuse & 0 & 0 & 0 & 0 & 0 & 0 & 0 & 0 & 0 & 6 \\
\hline Substance misuse, not alcohol & 0 & 0 & 0 & 0 & 0 & 0 & 1 & 1 & 0 & 16 \\
\hline Anxiety (including PTSD) & 0 & 0 & 0 & 2 & 1 & 0 & 0 & 0 & 0 & 19 \\
\hline Affective depression & 2 & 8 & 6 & 18 & 33 & 3 & 49 & 3 & 4 & 390 \\
\hline Affective bipolar & 2 & 1 & 2 & 14 & 3 & 4 & 18 & 2 & 5 & 195 \\
\hline Confusion/dementia & 5 & 12 & 29 & 97 & 70 & 5 & 202 & 2 & 8 & 557 \\
\hline Psychosis & 4 & 2 & 4 & 23 & 21 & 15 & 31 & 5 & 14 & 779 \\
\hline ADHD, Tourette & 0 & 0 & 0 & 0 & 0 & 1 & 3 & 3 & 1 & 187 \\
\hline Autism spectrum disorder, Asperger & 0 & 1 & 0 & 0 & 0 & 3 & 3 & 2 & 4 & 180 \\
\hline Head trauma & 0 & 0 & 0 & 0 & 2 & 2 & 4 & 0 & 0 & 12 \\
\hline Anorexia, eating disorder & 0 & 0 & 0 & 0 & 1 & 0 & 0 & 0 & 0 & 14 \\
\hline Other & 0 & 2 & 0 & 4 & 7 & 4 & 9 & 2 & 3 & 195 \\
\hline Other somatic/organic disease & 2 & 3 & 4 & 9 & 16 & 0 & 16 & 2 & 9 & 140 \\
\hline Total & 15 & 29 & 45 & 167 & 153 & 36 & 336 & 22 & 48 & 2690 \\
\hline
\end{tabular}

Total

15

$\begin{array}{llll}167 & 153 & 36 & 336\end{array}$

Note. This table includes patients sent for routine referrals or to exclude cerebral tumour $(N=2690)$. Results are based on information from the radiology information system. Values indicate number of scans. Suspect pathology includes changes that need further diagnostic workup to establish a diagnosis, e.g. suspected multiple sclerosis lesions. Other pathology includes different pathologies that do not fit into the other categories, e.g. post-operative changes, anomalies and changes secondary to trauma or suicide attempt. Infarcts includes both lacunar and cortical infarcts. Ventriculomegaly includes hydrocephalus and all other causes of increased ventricular size, including central atrophy. Tumours includes both benign and malign lesions, and metastases.

WMLS, white matter lesions; PTSD, post-traumatic stress disorder; ADHD, attention-deficit hyperactivity disorder 


\begin{tabular}{|c|c|c|c|c|c|c|c|c|c|c|}
\hline & \multicolumn{10}{|c|}{ Age, years } \\
\hline & $0-10$ & $11-20$ & $21-30$ & $31-40$ & $41-50$ & $51-60$ & $61-70$ & $71-80$ & $81-90$ & $91-100$ \\
\hline Pathologic scans & $15(6.9)$ & $43(7.1)$ & $18(5.2)$ & $29(12.8)$ & $34(16.5)$ & 66 (31.3) & $136(62.4)$ & $278(74.1)$ & $216(81.2)$ & $18(81.8)$ \\
\hline Haemorrhage & 0 & $1(0.2)$ & $1(0.3)$ & $1(0.4)$ & $3(1.4)$ & $3(1.4)$ & $1(0.5)$ & $4(1.1)$ & $1(0.4)$ & 0 \\
\hline Tumour & $1(0.5)$ & $1(0.2)$ & $2(0.6)$ & $2(0.9)$ & $1(0.5)$ & $6(2.8)$ & $7(3.2)$ & $6(1.6)$ & $3(1.1)$ & 0 \\
\hline Intra-axial tumour & $1(0.5)$ & 0 & $2(0.3)$ & $2(0.4)$ & 0 & $3(1.4)$ & $3(1.4)$ & $1(0.3)$ & 0 & 0 \\
\hline Ventriculomegaly & 0 & $3(0.5)$ & $1(0.3)$ & $2(0.9)$ & $2(1.0)$ & $3(1.4)$ & $4(1.8)$ & $17(4.5)$ & $11(4.1)$ & $2(9.1)$ \\
\hline Atrophy & $1(0.5)$ & $3(0.5)$ & $1(0.3)$ & $6(2.7)$ & $8(3.9)$ & $10(4.7)$ & $37(17.0)$ & $54(14.4)$ & $44(16.5)$ & $3(13.6)$ \\
\hline Infarcts & 0 & $4(0.7)$ & 0 & $3(1.3)$ & $3(1.4)$ & $9(4.3)$ & $23(10.6)$ & 57 (15.2) & $50(18.8)$ & $4(18.2)$ \\
\hline Cysts & $2(0.9)$ & $11(1.8)$ & $5(1.4)$ & $3(1.3)$ & $4(1.9)$ & $6(2.8)$ & $4(1.8)$ & $1(0.3)$ & 0 & 0 \\
\hline WMLS & $4(1.9)$ & $4(0.7)$ & $2(0.6)$ & $3(1.3)$ & $4(1.9)$ & $18(8.5)$ & $55(25.2)$ & 132 (35.2) & 105 (39.5) & $9(40.9)$ \\
\hline Suspect pathology & $2(0.9)$ & $7(1.2)$ & $3(0.9)$ & $4(1.8)$ & 0 & $1(0.5)$ & $1(0.5)$ & $3(0.8)$ & $1(0.4)$ & 0 \\
\hline Other pathology & $5(2.3)$ & 9 (1.5) & $3(0.9)$ & $5(2.2)$ & $9(4.4)$ & $9(4.3)$ & $4(1.8)$ & $4(1.1)$ & 0 & 0 \\
\hline Number of scans & 216 & 602 & 348 & 226 & 206 & 211 & 218 & 375 & 266 & 22 \\
\hline
\end{tabular}

those aged above 65 years, we found $77 \%$ pathology in patients with a mean age of 77 years. The confusion/dementia group showed the highest percentage, with $77 \%$ pathological scans. The populationbased Rotterdam scan study found that WMLs, meningiomas and infarcts are more prevalent in the older age groups. ${ }^{17}$ Illes et al. found that in research volunteers at a mean age of 75 years, $64 \%$ had pathological findings. ${ }^{16}$ Overall, and as expected, our cohort demonstrated an increased frequency of pathologies compared

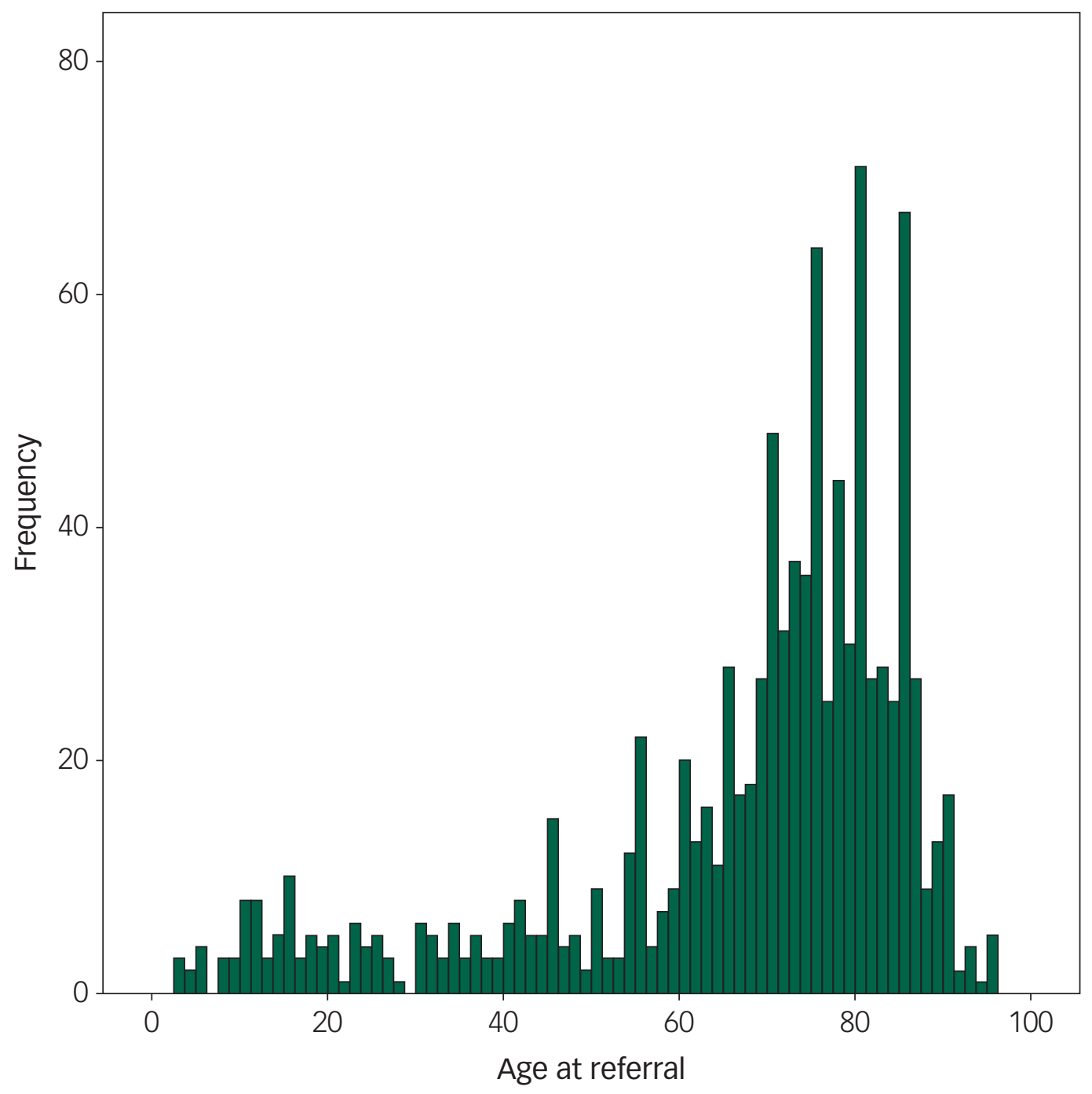

Fig. 1 The frequency of pathology at different ages in the population, showing the frequency of positive scans according to age. This figure includes only patients sent for routine referrals or to exclude cerebral tumour $(N=2690)$. We found that the frequency of pathology steeply increases by 65 years of age. 
with results from published cohorts of apparently healthy volunteers and healthy elderly participants.

We found that there are two age peaks in the referred patients, a younger age group (14-20 years) and an older age group (65-85 years). This bias in referrals may be caused by the debut of psychiatric symptoms and the need for diagnostic work-up in the younger age group, and perhaps by the need for a diagnostic work-up of memory problems and so forth in the older age group.

WMLs were the most common pathology overall, and it was the most common pathology in patients with depression, dementia and psychosis. Atrophy has been shown to be associated with psychosis. ${ }^{18}$ Atrophy in several cortical regions (e.g. cingulum, prefrontal and temporal) has also been demonstrated in patients with depression. ${ }^{19,20}$ Numerous studies have shown associations between latelife depression and WMLs. ${ }^{21}$ A recent review study found that WMLs are one of the main markers of vascular brain pathology. ${ }^{22}$ Thus, both neurodegeneration and vascular pathology are associated with depression. In patients with first-episode psychosis, the rate of WMLs was equal to that of healthy control participants. ${ }^{23}$ Few studies address the topic of WMLs in psychosis, but white matter deficits with diffusion tensor imaging has been shown both for mood disorders and psychosis. ${ }^{24}$ In patients with psychosis, we found $1.9 \%$ had cysts as the main pathology. In the literature, five case reports are published on the topic of arachnoid cysts in patients with psychosis ${ }^{25-29}$, but there are no cohort studies showing increased prevalence of arachnoid cysts in psychosis. In the neuroradiologic textbook by Osborne, arachnoid cyst prevalence is reported to be $1-2 \%$, indicating that our result does not represent an increased prevalence of arachnoid cysts in patients with psychosis.

In this study, we used the radiology reports to find and categorise the pathologies of the patients. This is a qualitative method, whereas many studies instead report quantitative results from analysing the actual images with volumetric methods, for example. From the literature, we have seen that both in bipolar disorder and schizophrenia, a higher incidence of ventriculomegaly and reduction in brain volume is reported. ${ }^{30-32}$ Atrophy is also commonly found in patients with depression or psychosis. In our cohort, we have a low number of reports of ventriculomegaly in the bipolar and psychosis groups (Table 3). In bipolar disorder and schizophrenia, the volumetric changes may be subtle and heterogeneous $^{30}$ and not easily picked up by the radiologist's eye. Studies of volumetric changes in psychiatric patients tend to be group analyses, whereas we study changes in individual patients. In individual patients, it may be impossible to detect such small changes. In ventricular size, there is a large variation in normal values in individual patients in the elderly population and overlapping values with disease (e.g. Alzheimer's disease ${ }^{33}$ ) and, to our knowledge, no good methods for visual assessment of ventricular enlargement in the adult population. This may explain the few reports of ventricular enlargement in patients with bipolar and patients with psychosis in our cohort.

However, in the diagnostic group of participants with the diagnosis confusion/dementia, we found higher reporting of atrophy than in the two abovementioned diagnostic groups. These patients tend to be older and one expects more atrophy in this group. This might have influenced the reporting. There are validated scales discerning changes related to normal aging versus changes such as atrophy that exceed what is expected for ageing, as described in the website Radiology Assistant (http://www.radiologyassistant.nl/ en/p43dbf6d16f98d/dementia-role-of-mri.html). In this article we have not reviewed the images, just the written reports, so we only had to register the pathology that was reported.

Characterisation into clinically relevant versus non-clinically relevant pathologies showed that $32.8 \%$ of our patients had clinically relevant pathologies, a result mainly driven by increased pathologies in the older age groups. Patients with psychosis had $14 \%$ clinically relevant pathologies, whereas patients with dementia had $75 \%$ clinically relevant pathologies. In a new publication from the population-based Nord-Trøndelag Health (HUNT) Study, Haberg et al. found $15.1 \%$ clinically relevant findings in a cohort of 1006 participants from the general population aged 50-66 years. ${ }^{34}$ Another MRI study of patients who presented with a psychotic episode (mean age of 31 years) reported $11.1 \%$ clinically relevant pathology. ${ }^{2}$ The most frequently reported pathology in our cohort was WMLs. WMLs are known to increase with increasing age, but they are also associated with medical conditions like dementia, ${ }^{35}$ depression ${ }^{36}$ and cerebrovascular disease. ${ }^{37}$

Neoplasms are potential causes of organic psychosis. The overall frequency $(1.1 \%)$ of neoplasms was higher in this selected clinical psychiatric population than reported in asymptomatic volunteers $(0.7 \%){ }^{38}$ The frequency of intra-axial tumours in this study was much higher in the group referred to exclude tumour (3.9\%) than in the group referred for routine scanning $(0.3 \%)$. The frequency of intra-axial tumours in the routine referral group is comparable with the frequency found by Katzman et al..$^{39}(0.2 \%)$ and Mueller et al. ${ }^{7}$ The clinical referral indication to exclude a brain tumour made it more likely to find a tumour, showing that the clinician makes a good distinction between the two indication types, and routine referrals for detection of brain tumours is not indicated.

During the 10-year study period, there was an increase in the number of referrals for brain scanning of patients from the division of psychiatry, and the number of MRI scans increased relative to computed tomography scans. Possible explanations for this are that there are more patients at psychiatric departments. This has been the case in another part of Norway in our study period. ${ }^{40}$ In our opinion, it is not unlikely that the development is also similar in Stavanger. The practice of referring patients with psychiatric disorders to brain scanning may also have changed either because of increased availability, ${ }^{41}$ generally more use of computed tomography scanning and MRI or the Norwegian guidelines recommending MRI early in the diagnostic work-up both in children and adults with psychosis. ${ }^{42}$ In contrast, the UK 2008 National Institute for Health and Care Excellence guidelines for psychosis and schizophrenia do not recommend routine scanning of patients with first episode of psychosis. ${ }^{43}$ We cannot conclude from this study which strategy is recommendable regarding routine referral of patients or not. Limited time for diagnostic work-up and time for observation in the hospital being replaced more and more by out-patient care may warrant such scanning to ensure correct diagnosis.

Among the study limitations were that we did not review the images but relied on the radiological report, as in a clinical setting. Our results are based on the quality of reporting on patient groups not included in a randomised study and are thus naturalistic. We do not know how the referring psychiatrist handled the pathological findings in these scans. Often, very little text was provided from the referring doctor about the clinical symptoms and findings. The categorisation of patients into different psychiatric diagnoses was done with limited clinical information available and is thus more uncertain than in a normal setting. Finally, we based our results only on the patients who were referred for scanning, however some patients may have not been referred, some did not come to their appointments and some did not manage to complete their scanning. This could introduce a bias in our results. A previous study by Mueller et al. ${ }^{7}$ showed that only $5 \%$ of psychiatric in-patients received a brain scan. Because our numbers are reported from both in- and out-patients, the percentage of referrals to imaging may be lower than the reported 5\%. The rate of referral may also be higher than $5 \%$ because Norwegian guidelines recommend imaging in the diagnostic work-up. 
Among the strengths of the study is the large number of both in- and out-patients from all clinics associated with the psychiatric division, investigated over a period of 10 years. Patients referred to a brain scan in the Stavanger region will preferably be referred to the radiology department, thus we believe that most of the patients from this area are included in our study, which makes it a representative cohort. We included all ages in our study, and as it is based on recent data, it is representative of current practice. The only similar imaging study was conducted in 1994-1998, with psychiatric inpatients aged $16-65$ years, and a smaller cohort. ${ }^{7}$

The practice of referring patients to brain scanning resulted in over $30 \%$ positive results. In children and young adults, $7 \%$ of scans had pathologic findings. This is lower than some reports on prevalence numbers of apparently healthy children. On the other hand, any positive scan may justify a liberal referral for brain scanning from a clinician's point of view. This is a topic that should be discussed further, and prospective studies may be needed to evaluate the indication for routine scanning in the youngest patients.

For adults and the elderly, the prevalence in our sample is increased compared with what has been reported in healthy populations and asymptomatic volunteers. From our results, routine scanning does not appear to reveal a higher prevalence of tumours than in the normal population, whereas clinical suspicion leads to detection of tumours at a higher rate.

Psychiatric patients are a group that may profit from better diagnostics and treatment of comorbid diseases. The lifespan of people with severe mental illness is shorter compared with the general population, ${ }^{44}$ with many illnesses such as schizophrenia showing high rates of cardiovascular morbidity and 10-25 years lower life expectancy. ${ }^{45}$ Increased awareness of WMLs or other brain pathologies secondary to brain scanning may lead to better diagnostic follow-up and treatment of comorbid disease in patients with a psychiatric disorder.

To conclude, although there is little pathology in the youngest age groups, the practice of MRI of the brain should at least be considered especially if there are specific neurological symptoms. For the adults and the elderly, we find increased pathologies compared with previous reports in healthy cohorts of the same age. Especially in the four groups affective depression, affective bipolar, confusion/ dementia and psychosis groups, more pathology is found than in the other diagnostic groups. These pathologies may lead to awareness and follow-up of comorbid diseases that might lead to increased life expectancy in the future. The use of MRI leads to less radiation to the patients and in most cases, better details of the pathology that exists. Further, we find an increased percentage of clinically relevant pathology in patients who had an MRI scan (37\%) compared with those who had computed tomography scans (30\%), which favours MRI scanning. If MRI is unavailable or impossible to perform, a brain computed tomography scan can be valuable.

Our study confirms the usefulness of having access to brain imaging in clinical psychiatry. Prospective studies to follow the clinical consequences of scanning and evaluating cost versus benefit of liberal versus strict referral practice are indicated.

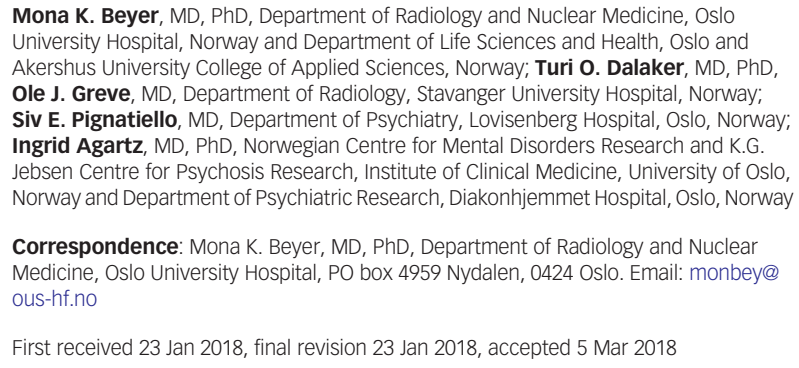

First received 23 Jan 2018, final revision 23 Jan 2018, accepted 5 Mar 2018

\section{Supplementary material}

Supplementary material is available online at https://doi.org/10.1192/bjo.2018.16

\section{Acknowledgements}

We thank Heidi Andersen Helse Vest, IKT and Kolbjorn S. Bronnick, PhD (Division of Psychiatry, Stavanger University Hospital) for their help. We also thank Andres S. Alonso, PhD and Wibeke Nordhøy, PhD (Department of Radiology and Nuclear Medicine, Oslo University Hospital) for reading through an early version of the manuscript.

\section{References}

1 Wahlund LO, Agartz I, Saaf J, Wetterberg L, Marions O. MRI in psychiatry: 731 cases. Psychiatry Res 1992; 45(2): 139-40.

2 Sommer IE, de Kort GA, Meijering AL, Dazzan P, Hulshoff Pol HE, Kahn RS, et al. How frequent are radiological abnormalities in patients with psychosis? A review of 1379 MRI scans. Schizophr Bull 2013; 39(4): 815-9.

3 Gupta S, Kanamalla U, Gupta V. Are incidental findings on brain magnetic resonance images in children merely incidental? J Child Neurol 2010; 25(12): 1511-6.

4 Wang L, Leonards CO, Sterzer P, Ebinger M. White matter lesions and depression: a systematic review and meta-analysis. J Psychiatr Res 2014; 56: 56-64.

5 Serafini G, Pompili M, Borgwardt S, Houenou J, Geoffroy PA, Jardri R, et al. Brain changes in early-onset bipolar and unipolar depressive disorders: a systematic review in children and adolescents. Eur Child Adolesc Psychiatry 2014; 23(11): 1023-41.

6 Boddaert N, Zilbovicius M, Philipe A, Robel L, Bourgeois M, Barthelemy C, et al. MRI findings in 77 children with non-syndromic autistic disorder. PLOS One 2009; 4(2): e4415.

7 Mueller C, Rufer M, Moergeli H, Bridler R. Brain imaging in psychiatry - a study of 435 psychiatric in-patients at a university clinic. Acta Psychiatr Scand 2006; 114(2): 91-100.

8 Haijma SV, Van Haren N, Cahn W, Koolschijn PC, Hulshoff Pol HE, Kahn RS. Brain volumes in schizophrenia: a meta-analysis in over 18000 subjects. Schizophr Bull 2013; 39(5): 1129-38.

9 Falkai P. Differential diagnosis in acute psychotic episode. Int Clin Psychopharmacol 1996; 11(Suppl 2): 13-7.

10 World Health Organization. Implementation of the International Statistical Classification of Diseases and related health problems, Tenth Revision (ICD-10). Epidemiol Bull 1997; 18(1): 1-4.

11 Kim BS, Illes J, Kaplan RT, Reiss A, Atlas SW. Incidental findings on pediatric MR images of the brain. AJNR Am J Neuroradiol 2002; 23(10): 1674-7.

12 Osborn AG. Osborn's BRAIN, Imaging, Pathology, and Anatomy. Amirsys, Inc, 2012.

13 George D, Mallery P. SPSS for Windows Step by Step: A Simple Guide and Reference. 11.0 update. Allyn \& Bacon, 2003.

14 Lubman DI, Velakoulis D, McGorry PD, Smith DJ, Brewer W, stuart G, et al. Incidental radiological findings on brain magnetic resonance imaging in firstepisode psychosis and chronic schizophrenia. Acta Psychiatr Scand 2002; 106(5): 331-6.

15 Gur RE, Kaltman D, Melhem ER, Ruparel K, Prabhakaran K, Riley M, et al. Incidental findings in youths volunteering for brain MRI research. AJNR Am J Neuroradiol 2013; 34(10): 2021-5.

16 Illes J, Rosen A, Huang L, Goldstein R, Raffin T, Swan G, et al. Ethical consideration of incidental findings on adult brain MRI in research. Neurology 2004; 62(6): 888-90.

17 Vernooij MW, Ikram MA, Tanghe HL, Vincent AJ, Hofman A, Krestin GP, et al. Incidental findings on brain MRI in the general population. N Engl J Med 2007; 357(18): 1821-8

18 van Lutterveld $R$, van den Heuvel MP, Diederen $K M$, de Weijer $A D$, Begemann MJ, Brouwer RM, et al. Cortical thickness in individuals with nonclinical and clinical psychotic symptoms. Brain 2014; 137(Pt 10): 2664-9.

19 Ribeiz SR, Duran F, Oliveira MC, Bezerra D, Castro CC, Steffens DC, et al. Structural brain changes as biomarkers and outcome predictors in patients with late-life depression: a cross-sectional and prospective study. PLOS One 2013; 8(11): e80049.

20 Mackin RS, Tosun D, Mueller SG, Lee JY, Insel P, Schuff N, et al. Patterns of reduced cortical thickness in late-life depression and relationship to psychotherapeutic response. Am J Geriatr Psychiatry 2013; 21(8): 794-802.

21 Tham MW, Woon PS, Sum MY, Lee TS, Sim K. White matter abnormalities in major depression: evidence from post-mortem, neuroimaging and genetic studies. J Affect Disord 2011; 132(1-2): 26-36. 
22 Kooistra M, Geerlings Ml, van der Graaf $\mathrm{Y}$, Mali WP, Vincken $\mathrm{KL}$, Kappelle $\mathrm{L}$, et al. Vascular brain lesions, brain atrophy, and cognitive decline. The Second Manifestations of ARTerial disease-Magnetic Resonance (SMART-MR) study. Neurobiol Aging 2014; 35(1): 35-41.

23 Zanetti MV, Schaufelberger MS, de Castro CC, Menezes PR, Scazufca M, McGuire PK, et al. White-matter hyperintensities in first-episode psychosis. Br J Psychiatry 2008; 193(1): 25-30.

24 Pridmore S, Bowe G. Neuroimaging in the field of psychoses. Malays J Med Sci 2011; 18(1): 6-11.

25 Baquero GA, Molero P, Pla J, Ortuno F. A schizophrenia-like psychotic disorder secondary to an arachnoid cyst remitted with neurosurgical treatment of the cyst. The open neuroimaging journal 2014; 8: 1-4.

26 Biswas PS, Sen D, Chaudhary S. Middle cranial fossa arachnoid cyst presenting with obsessive compulsive behaviour associated with psychosis - two cases. African journal of psychiatry 2012; 15(1): 59-60.

27 Kalapatapu RK. Aripiprazole treatment of psychosis in a child with posterior fossa retrocerebellar arachnoid cyst. Journal of child and adolescent psychopharmacology 2009; 19(3): 321-5.

28 Kuloglu M, Caykoylu A, Yilmaz E, Ekinci O. A left temporal lobe arachnoid cyst in a patient with schizophrenia-like psychosis: a case report. Progress in neuropsychopharmacology \& biological psychiatry 2008; 32(5): 1353-4.

29 Mironov A, John S, Auerbach J, Jamaleddine G. Acute onset of psychosis in patient with a left temporal lobe arachnoid cyst. Case reports in medicine 2014; 2014: 204025

30 Arnone D, Cavanagh J, Gerber D, Lawrie SM, Ebmeier KP, McIntosh AM Magnetic resonance imaging studies in bipolar disorder and schizophrenia: meta-analysis. Br J Psychiatry 2009; 195(3): 194-201.

31 Quigley SJ, Scanlon C, Kilmartin L, Emsell L, Langan C, Hallahan B, et al. Volume and shape analysis of subcortical brain structures and ventricles in euthymic bipolar I disorder. Psychiatry Res 2015; 233(3): 324-30.

32 Selvaraj S, Arnone D, Job D, Stanfield A, Farrow TF, Nugent AC, et al. Grey matter differences in bipolar disorder: a meta-analysis of voxel-based morphometry studies. Bipolar Disord 2012; 14(2): 135-45.

33 Brix MK, Westman E, Simmons A, Ringstad GA, Eide PK, Wagner-Larsen K, et al The Evans' Index revisited: New cut-off levels for use in radiological assessment of ventricular enlargement in the elderly. European journal of radiology 2017; 95: 28-32.

34 Haberg AK, Hammer TA, Kvistad KA, Rydland J, Muller TB, Eikenes L, et al. Incidental intracranial findings and their clinical impact; the HUNT MRI study in a general population of 1006 participants between $50-66$ years. PLOS One 2016; 11(3): e0151080.
35 Pendlebury ST, Rothwell PM. Prevalence, incidence, and factors associated with pre-stroke and post-stroke dementia: a systematic review and metaanalysis. Lancet Neurol 2009; 8(11): 1006-18.

36 Firbank MJ, Teodorczuk A, van der Flier WM, Gouw AA, Wallin A, Erkinjuntti T, et al. Relationship between progression of brain white matter changes and late-life depression: 3-year results from the LADIS study. $\mathrm{Br} J$ Psychiatry 2012; 201(1): 40-5.

37 Abraham HM, Wolfson L, Moscufo N, Guttmann CR, Kaplan RF, White WB. Cardiovascular risk factors and small vessel disease of the brain: blood pressure, white matter lesions, and functional decline in older persons. J Cereb Blood Flow Metab 2016; 36(1): 132-42.

38 Morris Z, Whiteley WN, Longstreth Jr WT, Weber F, Lee YC, Tsushima Y, et al. Incidental findings on brain magnetic resonance imaging: systematic review and meta-analysis. BMJ 2009; 339: b3016.

39 Katzman GL, Dagher AP, Patronas NJ. Incidental findings on brain magnetic resonance imaging from 1000 asymptomatic volunteers. JAMA 1999; 282(1): 36-9.

40 Togersen K, Bjerke E, Gjelstad K, Ruud T. Compulsory hospitalisation in menta health care in Ostfold in 2000 and 2010. Tidsskr Nor Laegeforen 2015; 135(1): 31-4.

41 Solheim $\mathrm{O}$, Torsteinsen $\mathrm{M}$, Johannesen TB, Jakola AS. Effects of cerebral magnetic resonance imaging in outpatients on observed incidence of intracranial tumors and patient survival: a national observational study. J Neurosurg 2014; 120(4): 827-32.

42 Norwegian Directorate of Health. Utredning, behandling og oppfølging av personer med psykoselidelser. 182. Helsedirektoratet/The Norwegian Directorate of Health, 2013.

43 National Institute for Health and Care Excellence. Structural Neuroimaging in First-Episode Psychosis. National Institute for Health and Care Excellence, 2008 (https://www.nice.org.uk/guidance/ta136).

44 de Hert M, Correll CU, Bobes J, Cetkovich-Bakmas M, Cohen D, Asai I, et al. Physical illness in patients with severe mental disorders. I. Prevalence, impact of medications and disparities in health care. World Psychiatry 2011; 10(1): 52-77

45 Dieset I, Andreassen OA, Haukvik UK. Somatic comorbidity in schizophrenia: some possible biological mechanisms across the life span. Schizophr Bull 2016; 42(6): 1316-19. 